Pacific Journal of Mathematics

THE PART METRIC IN CONVEX SETS

Bauer and Herbert Stanley Bear, J 


\title{
THE PART METRIC IN CONVEX SETS
}

\author{
Heinz Bauer and H. S. Bear
}

Any convex set $C$ without lines in a linear space $L$ can be decomposed into disjoint convex subsets (called parts) in a way which generalizes the idea of Gleason parts for a function space or function algebra. A metric $d$ (called part metric) can be defined on $C$ in a purely geometric way such that the parts of $C$ are the components in the $d$-topology. This paper treats the connection between the convex structure of $C$ and the metric $d$. The situation is particularly interesting when $C$ is closed with respect to a weak Hausdorff topology on $L$ (defined by a duality between $L$ and another linear space). Then $C$ is characterized by the set $C^{+}$of all continuous affine functions $F$ on $L$ satisfying $F(x) \geqq 0$ for all $x \in C$. This allows us to define $d$ in terms of the functions $\log F, F \in C^{+}$. Furthermore, $d$-completeness of $C$ can be derived from the completeness of $C$ in $L$. The "convexity" of the metric $d$ leads to the existence of a continuous selection function for lower semi-continuous mappings of a paracompact space into the nonempty $d$-closed convex subsets of one part of such a complete convex set $C$. We apply this result and the study of the part metric of the convex cone of positive Radon measures on a locally compact Hausdorff space to the problem of selecting in a continuous way mutually absolutely continuous representing measures for points in one part of a function space or function algebra.

1. The part metric and convex structure. We consider a real linear space $L$, and a convex set $C$ in $L$ which contains no whole line. We do not necessarily assume that $L$ has a topology.

The closed segment from $x$ to $y$ is denoted $[x, y]$. If $x, y \in C$, we say that $[x, y]$ extends (in $C$ ) by $r(>0)$ if $x+r(x-y) \in C$ and $y+r(y-x) \in C$. We write $x \sim y$ if $[x, y]$ extends by some $r>0$. It is shown in [1] that $\sim$ defines an equivalence relation in $C$.

The equivalence classes of $\sim$, called the parts of $C$, are clearly also convex. There is a metric $d$ on each part of $C$ defined by

$$
d(x, y)=\inf \left\{\log \left(1+\frac{1}{r}\right):[x, y] \text { extends by } r\right\}
$$

If $[x, y]$ extends by $r$ (in $C$ ), then $x+r^{\prime}(x-y)$ and $y+r^{\prime}(y-x)$ are in the part $\Pi$ of $x$ and $y$ for all $r^{\prime}<r$. It follows that one gets the same part metric on $I$ if one replaces $C$ by $I I$ in the definition of $d(x, y)$.

If $x \nsim y$, we write $d(x, y)=+\infty$. Then $d$ satisfies all axioms of 
a metric on $C$, except that it is not always finite. In spite of this, $d$ will be called the part metric on $C$. We could introduce min $(1, d)$ or $d / 1+d$ to obtain a bona fide metric on $C$ defining the same topology as $d$.

For each part $\Pi$ of $C$ and each $x \in \Pi$ we have

$$
\Pi=\{y \in C: d(x, y)<\infty\} .
$$

Therefore, the parts are open, and hence also closed.

The theorems of this sections establish connections between the part metric and the convex structure in $C$.

Theorem 1. Let $x, x^{\prime}, y, y^{\prime} \in C$ and $0 \leqq \lambda \leqq 1$. If $\left[x, x^{\prime}\right]$ and $\left[y, y^{\prime}\right]$ extend by $r$, then $\left[\lambda x+(1-\lambda) y, \lambda x^{\prime}+(1-\lambda) y^{\prime}\right]$ extends by $r$.

Proof. We have the identity

$$
\begin{gathered}
{[\lambda x+(1-\lambda) y]+r\left\{[\lambda x+(1-\lambda) y]-\left[\lambda x^{\prime}+(1-\lambda) y^{\prime}\right]\right\}} \\
=\lambda\left[x+r\left(x-x^{\prime}\right)\right]+(1-\lambda)\left[y+r\left(y-y^{\prime}\right)\right] .
\end{gathered}
$$

The term on the right is a convex combination of points which are in $C$ by hypothesis. The extension beyond $\lambda x^{\prime}+(1-\lambda) y^{\prime}$ follows by a symmetric argument. Notice that we do not assume that $x \sim y$ or $x^{\prime} \sim y^{\prime}$.

The corollaries below and Theorem 2 are basic to the proof of the selection theorem of $\S 6$. They are immediate consequences of Theorem 1.

COROLLARIES.

(i ) $d\left(\lambda x+(1-\lambda) y, \lambda x^{\prime}+(1-\lambda) y^{\prime}\right) \leqq \max \left[d\left(x, x^{\prime}\right), d\left(y, y^{\prime}\right)\right]$ for any points $x, x^{\prime}, y, y^{\prime} \in C$ and $0 \leqq \lambda \leqq 1$.

(ii) If $S$ is a convex subset of $C$ (not necessarily in one part) and $d(x, S)<\varepsilon, d(y, S)<\varepsilon$, then $d(\lambda x+(1-\lambda) y, S)<\varepsilon$ (even if $x \not y)$. (iii) $\{x: d(x, S)<\varepsilon\}$ is convex for any convex subset $S$ of $C$. In particular, d-balls are convex.

LEMma 1. If $u$ is a nonnegative concave function on $C$ (in particular an affine function) and $\Pi$ is a part of $C$, then either $u(x)>0$ for all $x \in \Pi$ or $u \equiv 0$ on $\Pi$.

Proof. If $x, y \in \Pi$ and the segment $[x, y]$ extends by $r$, then

$$
x_{0}=x+r(x-y) \in C \text {. }
$$




$$
x=\frac{1}{1+r} x_{0}+\frac{r}{1+r} y
$$

is a convex combination of $x_{0}$ and $y$. Since $u$ is concave and $\geqq 0$, this implies

$$
u(x) \geqq \frac{1}{1+r} u\left(x_{0}\right)+\frac{r}{1+r} u(y) \geqq \frac{r}{1+r} u(y) .
$$

Therefore it follows from $u(x)=0$ and $u(y) \geqq 0$ that $u(y)=0$.

THeORem 2. If $u$ is a nonnegative concave function on $C$, then $\log u$ is uniformly d-continuous on those parts where $u>0$. Consequently, all lower bounded concave functions on $C$ are d-continuous.

Proof. If $[x, y]$ extends by $r$, and $u>0$ on the part containing $x$ and $y$, then (1) shows that

$$
u(y) / u(x) \leqq 1+\frac{1}{r} .
$$

A symmetric argument shows that $u(x) / u(y) \leqq 1+(1 / r)$, and hence

$$
\begin{aligned}
& \left(1+\frac{1}{r}\right)^{-1} \leqq u(y) / u(x) \leqq 1+\frac{1}{r}, \\
& |\log u(x)-\log u(y)| \leqq \log \left(1+\frac{1}{r}\right) .
\end{aligned}
$$

Since $d(x, y)$ is the infimum of the right side,

$$
|\log u(x)-\log u(y)| \leqq d(x, y) .
$$

This proves the uniform continuity of $\log u$ on that part. The remaining assertion is evident.

Lemma 2. If $x, y \in C$ and $\phi(\lambda)=\lambda x+(1-\lambda) y$, then $\phi$ is $d$-continuous on $] 0,1[$. If $x \sim y$, then $\phi$ is even d-continuous on $[0,1]$.

Proof. The first assertion follows from the second, since for any two points $x \neq y$ in $C$ the open segment

$$
] x, y[=\{\lambda x+(1-\lambda) y: 0<\lambda<1\}
$$

lies in one part and since for $0<\lambda_{0}<1$ the point $\phi\left(\lambda_{0}\right)$ is a convex combination of points $x_{1}=\phi\left(\lambda_{1}\right)$ and $y_{1}=\phi\left(\lambda_{2}\right)$ where $0<\lambda_{1}<\lambda_{0}<\lambda_{2}<1$.

Assume therefore that $x \sim y$, and denote $\phi(\lambda)$ by $z_{\lambda}$ for $0 \leqq \lambda \leqq 1$. We will show that $d\left(z_{\lambda}, y\right) \rightarrow 0$ as $\lambda \rightarrow 0$. The left-side continuity of 
$\phi$ at a point $\left.\lambda_{0} \in\right] 0,1\left[\right.$ then follows from this by considering $\phi\left(\lambda_{0}\right)$ an end point, since

$$
\lambda x+(1-\lambda) y=\lambda^{\prime} x+\left(1-\lambda^{\prime}\right) \phi\left(\lambda_{0}\right)
$$

for some $\lambda^{\prime}=\lambda^{\prime}(\lambda)$ which tends to zero as $\lambda \rightarrow \lambda_{0}$ from the left. A symmetric argument gives continuity from the right. The following identities can easily be checked:

$$
\begin{aligned}
& y+r(y-x)=y+\frac{r}{\lambda}\left(y-z_{\lambda}\right) \\
& x+r(x-y)=z_{\lambda}+\frac{1-\lambda+r}{\lambda}\left(z_{\lambda}-y\right) .
\end{aligned}
$$

Therefore, if $[x, y]$ extends by $r$, then $\left[z_{\lambda}, y\right]$ extends by the minimum of the numbers $r / \lambda$, $(1-\lambda+r) / \lambda$, so $d\left(z_{\lambda}, y\right) \rightarrow 0$ as $\lambda \rightarrow 0$.

CoRollary. The parts of $C$ are connected, and are the components of $C$.

We next study the continuity of convex combinations.

TheOREM 3. For $x, y \in C$ and $0 \leqq \lambda \leqq 1$, denote by $\Psi(\lambda, x, y)$ the convex combination $\lambda x+(1-\lambda) y$. Then for each part $I I$ of $C$, the mapping

$$
\Psi:[0,1] \times \Pi \times \Pi \rightarrow \Pi
$$

is continuous. Moreover, the mapping

$$
\Psi:] 0,1[\times C \times C \rightarrow C
$$

is continuous.

Proof. Let $x, y, x_{0}, y_{0} \in C$, and $\lambda, \lambda_{0} \in[0,1]$. Then

$$
\begin{aligned}
d(\lambda x+ & \left.(1-\lambda) y, \lambda_{0} x_{0}+\left(1-\lambda_{0}\right) y_{0}\right) \\
\leqq & d\left(\lambda x+(1-\lambda) y, \lambda x_{0}+(1-\lambda) y_{0}\right. \\
& +d\left(\lambda x_{0}+(1-\lambda) y_{0}, \lambda_{0} x_{0}+\left(1-\lambda_{0}\right) y_{0}\right) .
\end{aligned}
$$

The first term on the right is dominated by $\max \left[d\left(x, x_{0}\right) d\left(y, y_{0}\right)\right]$ by Corollary (i) of Theorem 1. The behavior of the second term was studied in Lemma 2.

The next result concerns the description of the $d$-topology in a special case.

Let $C$ be an open convex set in a linear topological space and 
assume $C$ contains no line. Assume for convenience that $0 \in C$. Let $p$ be the Minkowski functional for $C: p(x)=\inf \{r: x \in r C, r>0\}$. Then $p$ is finite, subadditive and positive-homogeneous, and $C=\{x: p(x)<1\}$. If we let $q(x)=\max [p(x), p(-x)]$, then $q$ is absolutely homogeneous, and subadditive, and hence a seminorm. Since $C$ contains no line, $q$ is a norm. We will call $q$ the Minkowski norm for $C$.

THEOREM 4. If $C$ is an open convex set in a linear topological space, $0 \in C$, and $C$ contains no line, then $C$ has one part, and the part metric $d$ and the Minkowski norm define the same topology on $C$.

Proof. Since $C$ is open, it is clear that $C$ is one part. Let $x_{0} \in C$, so that $p\left(x_{0}\right)<1$. If $q\left(x-x_{0}\right)<1-p\left(x_{0}\right)$, then

$$
p(x)=p\left(x-x_{0}+x_{0}\right) \leqq p\left(x-x_{0}\right)+p\left(x_{0}\right) \leqq q\left(x-x_{0}\right)+p\left(x_{0}\right)<1 .
$$

That is, $x \in C$ if $q\left(x-x_{0}\right)<1-p\left(x_{0}\right)$. Since

$$
q\left[x_{0}+r\left(x_{0}-x\right)-x_{0}\right]=r q\left(x_{0}-x\right),
$$

$x_{0}+r\left(x_{0}-x\right) \in C$ if $r q\left(x_{0}-x\right)<1-p\left(x_{0}\right)$, or $r<\left(1-p\left(x_{0}\right)\right) / q\left(x_{0}-x\right)$. Also,

$$
q\left[x+r\left(x-x_{0}\right)-x_{0}\right]=(1+r) q\left(x-x_{0}\right),
$$

so that $x+r\left(x-x_{0}\right) \in C$ if $(1+r) q\left(x-x_{0}\right)<1-p\left(x_{0}\right)$, or

$$
q\left(x-x_{0}\right)<\left(1-p\left(x_{0}\right)\right) /(1+r) .
$$

Combining these facts, we get that $\left[x, x_{0}\right]$ extends by $r$ if

$$
1+r<\left(1-p\left(x_{0}\right)\right) / q\left(x-x_{0}\right) \quad\left(x \neq x_{0}\right) .
$$

If $q\left(x-x_{0}\right) \rightarrow 0$, then $\left[x, x_{0}\right]$ extends by arbitrarily large $r$, and hence $d\left(x, x_{0}\right) \rightarrow 0$.

Now suppose that $\left[x, x_{0}\right]$ extends by $r$, so that $p\left(x_{0}+r\left(x_{0}-x\right)\right)>1$ and $p\left(x+r\left(x-x_{0}\right)\right)<1$. Since $r\left(x_{0}-x\right)=x_{0}+r\left(x_{0}-x\right)-x_{0}$, we have

$$
\begin{aligned}
r p\left(x_{0}-x\right) & <p\left(x_{0}+r\left(x_{0}-x\right)\right)+p\left(-x_{0}\right) \\
& <1+p\left(-x_{0}\right) .
\end{aligned}
$$

Similarly, interchanging $x$ and $x_{0}$ gives

$$
r p\left(x-x_{0}\right)<1+p(-x) .
$$

Subadditivity of $p$ also gives

$$
p(-x) \leqq p\left(-x_{0}\right)+p\left(x_{0}-x\right) .
$$

Combining this last inequality and (2) gives 


$$
\begin{aligned}
r p\left(x-x_{0}\right) & <1+p\left(-x_{0}\right)+p\left(x_{0}-x\right) \\
& <1+p\left(-x_{0}\right)+\frac{1}{r}\left(1+p\left(-x_{0}\right)\right) \\
& =\left(1+\frac{1}{r}\right)\left(1+p\left(-x_{0}\right)\right) .
\end{aligned}
$$

The inequalities (2) and (3) show that $p\left(x_{0}-x\right)$ and $p\left(x-x_{0}\right)$ are both less than

$$
\frac{1}{r}\left(1+\frac{1}{r}\right)\left(1+p\left(-x_{0}\right)\right) \text {. }
$$

Consequently, $q\left(x-x_{0}\right) \rightarrow 0$ as $d\left(x, x_{0}\right) \rightarrow 0$ (i.e., $r \rightarrow \infty$ ).

Corollary. A bounded open convex set $C$ in a normed linear space $E$ has one part, and the part metric defines the same topology on $C$ as the given norm.

Proof. We may assume $0 \in C$. Then the Minkowski norm $q$ and the given norm are equivalent since $C$ contains some norm ball around 0 , and some norm ball contains $C \cap(-C)$ which is the ball $\{x: q(x)<1\}$. The result follows then from Theorem 4.

We remark that in the situation of the above corollary the part metric $d$ is in general not equivalent as a uniform structure to the norm metric. It suffices to assume that $E$ is the Euclidean space $R^{n}$. Then $C$ as an open set is not norm complete. But it will follow from Theorem 9 that $C$ as a part of the convex body $\bar{C}$ is $d$-complete.

Let us also point out that the above corollary together with Theorem 2 yields the well-known fact that a lower bounded concave function on a bounded open convex set in a normed linear space is continuous.

2. The part metric in a cone. In this section we give some additional properties of the equivalence relation $\sim$ and the part metric in the case that the convex set is a cone. We let $p$ denote a convex cone in the real linear space $L$ with vertex $0 \in P$, and assume that $P$ contains no line. We then have in particular no line in $P$ through 0, i.e., $P \cap(-P)=\{0\}$. Let $\leqq$ denote the partial order on $L$ induced by $P: x \leqq y$ means $y-x \in P$. Let us point out that $\{0\}$ is one part of $P$ since the vertex 0 is an extreme point of $P$.

Theorem 5. For any two points $x, y \in P$ the following statements hold:

(i ) $[x, y]$ extends by $r$ if and only if 


$$
\left(1+\frac{1}{r}\right) x \geqq y \quad \text { and } \quad\left(1+\frac{1}{r}\right) y \geqq x ;
$$

(ii) $[x, y]$ extends by $r$ if and only if $[p x, p y]$ extends by $r$ for a given number $p>0$; by $r$;

(iii) if $[x, y]$ extends by $r$ and $z \in p$, then $[x+z, y+z]$ extends

(iv) $x \sim p x$ for all $p>0$;

( v) if $x \sim y$, then $x \sim x+y$.

Proof. Condition (4) of (i) is just a restatement of the definition of extension in terms of the partial order. Note that transitivity of $\sim$ is immediate from (4). Conditions (ii) and (iii) also follow easily from (4). Condition (iv) is clear and (v) follows from (iii) and (iv) as follows:

$$
x+y \sim y+y=2 y \sim y \sim x .
$$

From (iv) and (v) we obtain

Corollary. Each part of $P$ is a convex subcone of $P$.

THEOREM 6. The part metric d of $P$ has the following properties:

(i) if $x \sim y$ and $x \neq 0, d(x, y)=\inf \{\log a: a x \geqq y$ and $a y \geqq x\}$;

(ii) $d(p x, p y)=d(x, y)$ for all $p>0$, all $x, y \in P$;

(iii) $d(x+z, y+z) \leqq d(x, y)$ for all $x, y, z \in P$;

(iv) $d(r x, s x)=|\log r-\log s|$ for $x \in P, x \neq 0, r>0, s>0$.

Proof. Conditions (i), (ii), (iii) follows from (i), (ii) and (iii) of Theorem 5; (iv) follows from (i) by observing that $d(r x, s x)=d\{(r / s) x, x\}$ because of (ii).

THEOREM 7. The mappings $(\lambda, x) \rightarrow \lambda x$ from $] 0,+\infty[\times p$ into $P$ and $(x, y) \rightarrow x+y$ from $P \times P$ into $P$ are continuous (with respect to the part metric $d$ on $P$ ). Explicitly,

$$
\begin{gathered}
d\left(\lambda x, \lambda x_{0}\right) \leqq d\left(x, x_{0}\right)+\left|\log \lambda-\log \lambda_{0}\right| \\
\quad\left(x, x_{0} \in P, x_{0} \neq 0, \lambda>0, \lambda_{0}>0\right) ; \\
d\left(x+y, x_{0}+y_{0}\right) \leqq \max \left[d\left(x, x_{0}\right), d\left(y, y_{0}\right)\right] \quad\left(x, y, x_{0}, y_{0} \in P\right) .
\end{gathered}
$$

Proof. Using the triangle inequality and Theorem 6, (ii), (iv), we have

$$
\begin{aligned}
d\left(\lambda x, \lambda_{0} x_{0}\right) & \leqq d\left(\lambda x, \lambda x_{0}\right)+d\left(\lambda x_{0}, \lambda_{0} x_{0}\right) \\
& =d\left(x, x_{0}\right)+\left|\log \lambda-\log \lambda_{0}\right| .
\end{aligned}
$$


From Corollary (i) of Theorem 1 we have

$$
d\left(\frac{1}{2} x+\frac{1}{2} y, \frac{1}{2} x_{0}+\frac{1}{2} y_{0}\right) \leqq \max \left[d\left(x, x_{0}\right), d\left(y, y_{0}\right)\right] \text {. }
$$

Multiplying both elements in the left term by 2 preserves distance (Theorem 6, (ii)) and gives (6). The continuity of $(\lambda, x) \rightarrow \lambda x$ at a point $\left(\lambda_{0}, 0\right), \lambda_{0}>0$, is evident since $\{0\}$ is a part, and hence open in $P$.

3. Completeness of part metric. In this section we give some conditions which imply the completeness of the part metric. We begin with a functional analytic description of this metric.

Following Choquet [7], and Bourbaki [4] we will say that a linear space $L$ is a weak space if it is endowed with a weak topology $\sigma(L, M)$ derived from a duality between $L$ and some linear space $M$. Hence there is a bilinear form $(x, y) \rightarrow\langle x, y\rangle$ on $L \times M$, and $\sigma(L, M)$ is the weakest topology on $L$ making all linear functionals $x \rightarrow\langle x, y\rangle, y \in M$, continuous. Remember that $\sigma(L, M)$ is then a locally convex topology on $L$. We will always assume that the weak space $L$ is Hausdorff. This is equivalent to the fact that the linear forms $x \rightarrow\langle x, y\rangle$ with $y \in M$ separate the points of $L$.

A set $C$ in a weak space $L$ will be called complete if it is complete in the uniform structure induced in $C$ by the underlying topology $\sigma(L, M)$. Obviously, each complete set in a weak Hausdorff space $L$ is also closed.

Each closed convex subset $C$ of a weak Hausdorff space $L$ is an intersection of closed half-spaces. Therefore, we have

$$
C=\bigcap_{F \in C^{+}}\{x \in L: F(x) \geqq 0\}
$$

where $C^{+}$denotes the set of all continuous affine functions on $L$ which are nonnegative on $C$. That is $C^{+}$characterizes $C$ in the sense that $x \in C$ if and only if $F(x) \geqq 0$ for all $F \in C^{+}$. Remember [4; p. 91] that each continuous affine function $F$ on $L$ is of the form $F(x)=\left\langle x, y_{F}\right\rangle+$ constant with $y_{F} \in M$.

THEOREM 8. If $C$ is a closed convex set containing no line in a weak Hausdorff space, then

$$
d(x, y)=\sup \left\{|\log F(x)-\log F(y)|: F \in C^{+}, F(y)>0\right\}^{1}
$$

for all points $x, y \in C$.

Proof. Assume first that $x \sim y$. The condition $F(y)>0$ is then equivalent to assuming that $F>0$ on the part containing $y$ by Lemma 1 .

\footnotetext{
${ }^{1}$ We make use of the convention: $\log 0=-\infty$.
} 
Since $C^{+}$characterizes $C$, we have $x+r(x-y)=(1+r) x-r y \in C$ if and only if

$$
\left(1+\frac{1}{r}\right) F(x) \geqq F(y)
$$

for all $F \in C^{+}$. Similarly, $y+r(y-x) \in C$ if and only if $\{1+(1 / r)\} F(y) \geqq$ $F(x)$ for all $F \in C^{+}$. Hence $[x, y]$ extends by $r$ if and only if

$$
\left(1+\frac{1}{r}\right)^{-1} \leqq F(x) / F(y) \leqq 1+\frac{1}{r}
$$

for all $F \in C^{+}$with $F(y)>0$. Hence $[x, y]$ extends by $r$ if and only if

$$
|\log F(x)-\log F(y)| \leqq \log \left(1+\frac{1}{r}\right)
$$

for all $F \in C^{+}$with $F(y)>0$. Therefore $d(x, y)$, which is the infimum of the right side, also is the supremum of the left side.

In the case $x \ngtr y$ we have to show that the supremum in (7) is infinite. We can assume specifically that the segment $[x, y]$ does not extend beyond $y$. Hence $y+\varepsilon(y-x) \notin C$ for each $\varepsilon>0$. Therefore, for fixed $\varepsilon>0$, there exists a function $F \in C^{+}$such that

$$
(1+\varepsilon) F(y)-\varepsilon F(x)=F(y+\varepsilon(y-x))<0 .
$$

From this follows that $F(x)>0$ and

$$
|\log F(x)-\log F(y)|=\log F(x)-\log F(y)>\log \left(1+\frac{1}{\varepsilon}\right) .
$$

But $\log \{1+(1 / \varepsilon)\} \rightarrow+\infty$ as $\varepsilon \rightarrow 0$. If $F(y)=0$, we can replace $F$ by $F+\alpha$, where $\alpha$ is a small positive number and draw the same conclusion.

Corollary ( i ). If $C$ is a closed convex set containing no line in a weak Hausdorff space, then a fundamental system of $d$-neighborhoods of a point $x_{0} \in C$ is given by the sets

$$
\begin{aligned}
& \left\{x \in C:\left|F(x)-F\left(x_{0}\right)\right|<\varepsilon, \quad \text { all } F \in C^{+}, F\left(x_{0}\right)=1\right\} \\
= & \left\{x \in C:\left|F(x)-F\left(x_{0}\right)\right|<\varepsilon, \text { all } F \in C^{+}, 0<F\left(x_{0}\right) \leqq 1\right\},
\end{aligned}
$$

where $\varepsilon$ ranges over the positive numbers.

Proof. We first prove that the sets in (8) are the same for given $\varepsilon>0$. Clearly, the second set is contained in the first one. Conversely, if $0<F\left(x_{0}\right)=\alpha \leqq 1$ for $F \in C^{+}$, then 


$$
\frac{1}{\alpha} F\left(x_{0}\right)=1 \text { and }\left|F(x)-F\left(x_{0}\right)\right| \leqq\left|\frac{1}{\alpha} F(x)-\frac{1}{\alpha} F\left(x_{0}\right)\right| .
$$

By Theorem 8 we know that a sequence $\left\{x_{n}\right\}$ in $C$ is $d$-convergent to $x_{0} \in C$ if and only if $\log F\left(x_{0}\right) \rightarrow \log F(x)$ uniformly for all $F \in C^{+}$ satisfying $F\left(x_{0}\right)>0$. Since

$$
\log F\left(x_{n}\right)-\log F\left(x_{0}\right)=\log \left[F\left(x_{n}\right) / F\left(x_{0}\right)\right]
$$

does not change if $F$ is multiplied by a positive constant, $d\left(x_{n}, x_{0}\right) \rightarrow 0$ is equivalent to $F\left(x_{n}\right) \rightarrow F\left(x_{0}\right)$ uniformly for all $F \in C^{+}$with $F\left(x_{0}\right)=1$.

Corollary (ii). (cf. [10, Lemma 1.2]) If $C$ is a closed convex set containing no line in a weak Hausdorff space, then the d-topology is the weakest topology such that for each $x_{0} \in C$ the set $\left\{F \in C^{+}: F\left(x_{0}\right)=1\right\}$ is equicontinuous.

In what follows we will assume that $C$ is a complete convex set without lines in a weak Hausdorff space $L$. Then a result of Choquet [7, Corollary 1, p. 1908] states that each continuous affine function on $L$ is the difference of two functions in $C^{+}$. This result will be fundamental for our proofs.

Corollary (iii). Let $C$ be a complete convex set containing no line in a weak Hausdorff space L. Then the part metric topology is stronger than the topology of $C$.

Proof. By the result of Choquet mentioned above the topology of $L$ is induced by the set $C^{+}$. Therefore, for any point $x_{0} \in C$ exist arbitrary small $L$-neighborhoods $W$ of $x_{0}$ of the form

$$
W=\left\{x \in C:\left|F_{i}(x)-F_{i}\left(x_{0}\right)\right|<\varepsilon, F_{i} \in C^{+}, i=1, \cdots, n\right\},
$$

where we may assume $F_{i}\left(x_{0}\right)>0$ for all $i=1, \cdots, n$. We find a smaller $d$-neighborhood of $x_{0}$ as follows: Let $F_{\imath}^{\prime}=F_{i} / F_{i}\left(x_{0}\right)$ so that $F_{i}^{\prime}\left(x_{0}\right)=1$ and $\left|F_{i}^{\prime}(x)-F_{i}^{\prime}\left(x_{0}\right)\right|<\varepsilon^{\prime}$ with $\varepsilon^{\prime}=\min \left[\varepsilon / F_{i}\left(x_{0}\right), \cdots, \varepsilon / F_{n}\left(x_{0}\right)\right]$. Then

$$
W_{0}=\left\{x \in C:\left|F(x)-F\left(x_{0}\right)\right|<\varepsilon^{\prime}, F \in C^{+}, F\left(x_{0}\right)=1\right\}
$$

is a $d$-neighborhood of $x_{0}$, and clearly $W_{0} \subset W$.

THEOREM 9. If $C$ is a complete convex set containing no line in a weak Hausdorff space $L$, then $C$ is $d$-complete and each part of $C$ is d-complete.

Proof. Again by the result of Choquet we can use the fact that the topology on $L$ is that induced by the set $C^{+}$of all continuous 
affine functions on $L$ which are nonnegative on $C$. Let $\left\{x_{n}\right\}$ be a $d$ Cauchy sequence in $C$. Then all $x_{n}$ are in one part. Let $C_{0}^{+}$be the functions $C^{+}$which are strictly positive on this part. By Theorem 8, $\left\{\log F\left(x_{n}\right)\right\}$ is a Cauchy sequence uniformly for $F \in C_{0}^{+}$. For each fixed $F \in C^{+},\left\{F\left(x_{n}\right)\right\}$ is therefore a Cauchy sequence. Since $C$ is complete there is $x_{0} \in C$ such that $F\left(x_{n}\right) \rightarrow F\left(x_{0}\right)$ for each $F \in C^{+}$. Since $\left\{x_{n}\right\}$ is $d$-Cauchy, this convergence is uniform in those $F$ such that $F\left(x_{0}\right)=1$. Therefore $d\left(x_{n}, x\right) \rightarrow 0$, and $C$ is $d$-complete. Then also each part is $d$-complete as a $d$-closed subset of $C$.

We next treat the special case where our convex set $C$ is a convex cone $P$ with vertex $0 \in P$. In this case Theorem 8 can be restated in a somewhat different form.

THEOREM 8'. Let $P$ be a closed convex cone with vertex $0 \in P$ containing no line in a weak Hausdorff space $L$, and denote by $P_{+}$the set of all continuous linear forms $f$ which are $\geqq 0$ on $P$. Then

$\left(8^{\prime}\right) d(x, y)=\sup \left\{|\log f(x)-\log f(y)|: f \in P_{+}, f(x)>0 f(y)>0\right\}$

for all points $x, y \in P$.

Proof. $P_{+}$characterizes $P$ in the sense

$$
P=\bigcap_{f \in P_{+}}\{x \in L: f(x) \geqq 0\} .
$$

This is well-known and can be deduced from (7) by remarking that each continuous affine function $F$ on $L$ satisfying $F \geqq 0$ on $P$ is of the form $F=f+\alpha$ where $f \in P_{+}$and $\alpha \geqq 0$. But with this the proof of Theorem 8 can be repeated by replacing $C$ by $P$ and $C^{+}$by $P_{+}$. Only the last sentence of the proof has to be dropped.

REMARK. If $x$ and $y$ are in the same part $\Pi$ of $P$ the condition $f(x)>0$ or $f(y)>0$ is equivalent to $f(y)>0$ and even to $f>0$ on $\Pi$. Therefore the sets

$$
\left\{x \in \Pi:\left|f(x)-f\left(x_{0}\right)\right|<\varepsilon, \text { all } f \in P_{+}, 0 \leqq f\left(x_{0}\right) \leqq 1\right\}
$$

for $\varepsilon>0$ form a fundamental system of $d$-neighborhoods of $x_{0} \in \Pi$ in II (cf. Theorem 8, Corollary (i)).

Let us point out that for a closed convex cone it is easy to check whether it contains a line:

Lemma 3. Let $P$ be a closed convex cone with vertex $0 \in P$ in a weak Hausdorff space. $P$ contains no line if and only if $P \cap(-P)=\{0\}$. 
Proof. The condition $P \cap(-P)=\{0\}$ is necessary since it says that $P$ does not contain a line through the origin. To prove its sufficiency, assume that $x+t(y-x) \in P$ for all real numbers $t$. Since $P_{+}$characterizes $P$ this is equivalent to $f(x)+t f(y-x) \geqq 0$ for all $t$ and all $f \in P_{+}$. Therefore, $f(y-x)=0$ for all $f \in P_{+}$; i.e., $y-x \in P \cap(-P)$ and hence $x=y$.

The next theorem gives a completeness criterion for the part metric in a cone. Remember that a convex subset $K$ of a convex cone $P$ with vertex $0 \in P$ is called a section or base for $P$ if each ray

$$
\{r x: r>0\}, x \in P, x \neq 0,
$$

intersects $K$ exactly once. Hence $P=\{r x: x \in K, r \geqq 0\}$ if $K$ is a section of $P$.

THEOREM 10. Let $P$ be a convex cone satisfying $P \cap(-P)=\{0\}$ in a weak Hausdorff space $L$. If $P$ has a compact section $K$ then $P$ and hence also $P \backslash\{0\}$ are d-complete.

Proof. It is well-known [4, p. 113] that $P$ is complete in $L$. Therefore, by the above lemma $P$ does not contain any line. The result follows then from Theorem 9 , since $P \backslash\{0\}$ is a part of $P$.

Let us remark that for a convex cone $P$ with a section $K$ and without lines the parts of $K$ are the intersections of the parts of $P$ with $K$. This results from the observation that by the definition of a section a segment $[x, y] \subset K$ extends by $r$ in $K$ if and only if it extends by $r$ in $P$. Hence also the part metric of $K$ is the restriction of the part metric in $P$.

Theorem 10 will be applied in particular to the following situation:

CoROllary. Let $B$ be a linear space of real continuous functions on a compact Hausdorff space containing the constant functions. Let $L=B^{\prime}$ be the topological dual of $B$ viewed as a normed space with the norm of uniform convergence. Then $P=\left\{F \in B^{\prime}: F \geqq 0\right\}$ and $K=$ $\{F \in P: F(1)=1\}$ are $d$-complete.

It suffices to remark that $K$ is compact in the weak topology $\sigma\left(L^{\prime}, L\right)$ and that $K$ is a section of the cone $P$.

4. The space of Radon measures. Let $\mathscr{C}=\mathscr{M}(X)$ be the space of all real Radon measures on a locally compact Hausdorff space $X$ with the vague topology. ${ }^{2}$ This is the weak (Hausdorff) topology $\sigma(\mathscr{M}, \mathscr{K})$

${ }^{2}$ We follow Bourbaki [5], [6] in terminology and notation for integration theory. 
induced by the linear space $\mathscr{K}=\mathscr{K}(X)$ of all continuous real-valued functions on $X$ with compact support. The convex cone $\mathscr{C}_{+}=\mathscr{C}_{+}(X)$ of all measures $\mu \geqq 0$ in $\mathscr{C}$ is then complete; it also satisfies $\mathscr{C l}_{+} \cap\left(-\mathscr{L}_{+}\right)=\{0\}$, and hence does contain any line by Lemma 3 . Therefore by Theorem $9, \mathscr{C}_{+}$is complete with respect to the part metric $d$ on $\mathscr{C}_{+}$. In this section we will describe the parts of $\mathscr{C}_{+}$ and the metric $d$.

By Theorem 5 the equivalence relation $\sim$ in $\mathscr{C}_{+}$is defined as follows: $\mu \sim \nu$ if and only if $\alpha \mu \geqq \nu$ and $\alpha \nu \geqq \mu$ for some number $\alpha>0$ (necessarily $\alpha \geqq 1$ if $\mu \neq 0$ or $\nu \neq 0$ ). In particular the zero measure is a part by itself. Consequently [6; p. 51] two measures $\mu, \nu \in \mathscr{C}_{+}$are equivalent if and only if they are mutually absolutely continuous, with (locally integrable) Radon-Nikodym derivatives which locally almost everywhere are bounded and bounded below by some number $\alpha>0$. Hence the parts of $\mathscr{C}_{+}$can be described as follows: for any fixed $\mu \in \mathscr{C}_{+}$, consider the set $P_{\mu}$ of all "functions" $g \in L^{\infty}(\mu)$ which are positive and bounded away from zero. Then the part $\Pi_{\mu}$ of $\mathscr{l l}_{+}$containing $\mu$ is the set

$$
\Pi_{\mu}=\left\{g \mu: g \in P_{\mu}\right\} \text {. }
$$

Therefore, we can transfer the $L^{\infty}(\mu)$-metric to $\Pi_{\mu}$ via the bijection $g \rightarrow g \mu$ of $P_{\mu}$ onto $\Pi_{\mu}$.

The following result describes the topology derived from $d$ in a part, thus generalizing and improving Theorem 3 of [2].

Theorem 11. The part metric $d$ in each part $\Pi_{\mu}$ of $\mathscr{L}_{+}(X)$ is topologically equivalent to the $L^{\infty}(\mu)$-metric on the corresponding functions of $P_{\mu}\left(\mu \in \mathscr{C}_{+}(X)\right)$.

Proof. Let $g_{0}, g_{1}, g_{2}, \cdots$ be a sequence in $P_{\mu}$. We have to prove

$$
\lim _{n \rightarrow \infty}\left\|g_{n}-g_{0}\right\|_{\infty}=0 \text { if and only if } \lim _{n \rightarrow \infty} d\left(g_{n} \mu, g_{0} \mu\right)=0 \text {. }
$$

By a well-known result on $L^{p}$-spaces (cf. Bourbaki [5, pp. 211-213]), one has

$$
\left\|g_{n}-g_{0}\right\|_{\infty}=\sup \left|\int v\left(g_{n}-g_{0}\right) d \mu\right|
$$

where the supremum is taken over all functions $v \in \mathscr{K}$ satisfying $\int|v| d \mu \leqq 1$. On the other hand, from Theorem $8^{\prime}$ and the remark following this theorem, we have $\lim _{n \rightarrow \infty} d\left(g_{n} \mu, g_{0} \mu\right)=0$ if and only if

$$
\lim _{n \rightarrow \infty} \int u\left(g_{n}-g_{0}\right) d \mu=0
$$


holds uniformly for all functions $u \in \mathscr{K}_{+}$satisfying $\int u g_{0} d \mu \leqq 1$. From this fact and the following two simple observations the theorem follows: (i) for each function $u \in \mathscr{L}_{+}$satisfying $\int u g_{0} d \mu \leqq 1$, one has

$$
\int \alpha u d \mu \leqq \int u g_{0} d \mu \leqq 1
$$

where $\alpha>0$ is locally almost everywhere a lower bound for $g_{0}$. For each function $v \in \mathscr{K}$ satisfying $\int|v| d \mu \leqq 1$ one has

$$
\int|v| g_{0} d \mu \leqq\left\|g_{0}\right\|_{\infty} \int|v| d \mu \leqq\left\|g_{0}\right\|_{\infty} \text { and }|v| \in \mathscr{K}_{+} \text {. }
$$

5. A selection theorem for the part metric. In this section we prove a generalization of the following theorem of $\mathrm{E}$. Michael [11, Th. 1], [12, Th. 3.2"]:

Theorem M. If $T$ is a paracompact space and $\phi$ is a lower semicontinuous function from $T$ to the nonempty closed convex subsets of a Banach space $Y$ then there exists a continuous selection function $f: T \rightarrow Y$ such that $f(t) \in \dot{\phi}(t)$ for all $t \in T$.

In our version of Theorem $M$ we replace the Banach space $Y$ by a convex set with a complete metric which is convex in the sense of the following definition.

Definition. Let $Y$ be a convex set in a linear space. A metric $d$ on $Y$ will be called convex if

(i) the mapping $(\lambda, x, y) \rightarrow \lambda x+(1-\lambda) y$ of $[0,1] \times Y \times Y$ into $Y$ is continuous, and if

(ii) the set $\{y \in Y: d(y, S)<\varepsilon\}$ is convex for each $\varepsilon>0$ and each convex subset $S$ of $Y$.

Theorem $\mathrm{M}$ follows from our result by the remark that the norm metric on a Banach space $Y$ is convex and complete. From Theorem 1 , Corollary (iii) and Theorem 3 it is clear that the part metric of a convex set $C$ without any line is convex on each part $\Pi$ of $C$.

We recall that a mapping $\phi$ from a topological space $T$ to the subsets of a topological space $Y$ is called lower semi-continuous (l.s.c.) if for any open set $U \subset Y$, the set of all $t \in T$ such that $\phi(t) \cap U \neq \varnothing$ is open. If $\phi$ is the inverse of a mapping $R$ from $Y$ onto $T$, then it is easy to see that $\phi$ is l.s.c. if and only if $R$ is an open mapping. 
For the proof of our selection theorem the same kind of arguments can be used as in [11].

Lemma 4. Let $T$ be a paracompact space, $Y$ a convex set with a convex metric $d$, and let $\phi$ be a lower semi-continuous function from $T$ to the nonempty convex subsets of $Y$. Then for any $r>0$, there is a d-continuous function $f: T \rightarrow Y$ such that

$$
d(f(t), \dot{\phi}(t))<r \text { for all } t \in T .
$$

Proof. For $y \in Y$, let $U_{y}=\{t \in T: d(y, \phi(t))<r\}$; that is, $U_{y}$ is the open set of points $t \in T$ such that $\phi(t)$ intersects the open $r$-ball around $y$. The family $\left(U_{y}\right)_{y \in Y}$ covers $T$ since $t \in U_{y}$ if $y \in \dot{\phi}(t) \neq \varnothing$. The space $T$ being paracompact, there exists an open locally finite refinement $\left(V_{\alpha}\right)_{\alpha \in A}$ of the covering $\left(U_{y}\right)$. Thus each $V_{\alpha}$ is contained in some $U_{y}$, and each $t \in T$ has a neighborhood which intersects only a finite number of $V_{\alpha}$. Let $\left(P_{\alpha}\right)_{\alpha \in A}$ be a partition of unity subordinate to $\left(V_{\alpha}\right)_{\alpha \in A}$; i.e., $p_{\alpha}$ is a nonnegative continuous function on $T, p_{\alpha}=0$ off $V_{\alpha}$, and $\Sigma p_{\alpha}(t)=1$ for all $t \in T$. For each $\alpha \in A$, pick $y_{\alpha} \in Y$ so that $V_{\alpha} \subset U_{y_{\alpha}}$. Let the function $f: T \rightarrow Y$ be defined by $f(t)=$ $\sum_{\alpha \in A} p_{\alpha}(t) y_{\alpha}, t \in T$. For each $t_{0} \in T$, there is a neighborhood $N_{t_{0}}$ which intersects only a finite number of $V_{\alpha}$, so that $p_{\alpha}=0$ on $N_{t_{0}}$ for all but a finite number of $\alpha$. Thus in $N_{t_{0}}$ the sum $f(t)$ is a convex combination of a finite number of $y_{\alpha} \in Y$ :

$$
f(t)=p_{\alpha_{1}}(t) y_{\alpha_{1}}+\cdots+p_{\alpha_{n}}(t) y_{\alpha_{n}} \quad\left(t \in N_{t_{0}}\right) .
$$

Thus because of the convexity of the metric $f$ is $d$-continuous on each $N_{t_{0}}$, hence on $T$. Furthermore, $f(t) \in Y$ is a convex combination of points $y_{\alpha}$ such that $p_{\alpha}(t) \neq 0$, hence such that $t \in V_{\alpha} \subset U_{y_{\alpha}}$. That is all these $y_{\alpha}$ are within a distance $r$ of $\phi(t)$. Hence $d(f(t), \phi(t))<r$ since each of the sets $\{y \in Y: d(y, \phi(t)<r\}$ is convex.

Theorem 12. Let $T$ be a paracompact space and $Y$ a convex set with a complete convex metric $d$. If $\phi$ is a lower semi-continuous mapping from $T$ to the nonempty closed convex subsets of $Y$, then there exists a continuous function $f: T \rightarrow Y$ such that $f(t) \in \phi(t)$ for all $t \in T$.

Proof. Michael's proof [11, p. 235] for a Banach space works without change in view of the preceding lemma. We repeat the argument here for the reader's convenience.

We construct inductively a sequence $f_{n}: T \rightarrow Y$ of continuous mappings such that uniformly for all $t \in T$ 


$$
\begin{aligned}
& d\left(f_{n}(t), f_{n+1}(t)\right)<1 / 2^{n-1} \\
& d\left(f_{n}(t), \phi(t)\right)<1 / 2^{n} .
\end{aligned} \quad(n=1,2, \cdots) .
$$

Then by condition $(10)\left(f_{n}\right)$ is uniformly Cauchy, and hence by the completeness of $Y$ converges to a continuous mapping $f: T \rightarrow Y$. The condition (11) insures $f(t) \in \phi(t)$ since each $\phi(t)$ is closed.

The preceding lemma proves the existence of a mapping $f_{1}: T \rightarrow Y$ such that $d\left(f_{1}(t), \phi(t)\right)<1 / 2$ for all $t \in T$. Suppose $f_{1}, \cdots, f_{n}$ have been defined satisfying (10) and (11). Define for each $t \in T$

$$
\phi_{n+1}(t)=\phi(t) \cap\left\{y \in Y: d\left(y, f_{n}(t)<1 / 2^{n}\right\} .\right.
$$

Then $\phi_{n+1}(t) \neq \varnothing$ by (10). Since the metric $d$ is convex, $\phi_{n+1}(t)$ is the intersection of two convex sets and therefore convex. We show that $\phi_{n+1}$ is 1.s.c., and then appeal to the lemma for the existence of $f_{n+1}$ such that $d\left(f_{n+1}(t), \phi_{n+1}(t)\right)<1 / 2^{n+1}(t \in T)$. This implies then

$$
d\left(f_{n}(t), f_{n+1}(t)\right)<1 / 2^{n}+1 / 2^{n+1}<1 / 2^{n-1}
$$

as required in (10).

To show that $\phi_{n+1}$ is 1.s.c., suppose $\phi_{n+1}\left(t_{0}\right) \cap U \neq \varnothing$ for some open set $U \subset Y$ and some $t_{0} \in T$. For each positive $\varepsilon<1 / 2^{n}$, there is a point $y_{0} \in \phi\left(t_{0}\right) \cap U$ such that $d\left(y_{0}, f_{n}\left(t_{0}\right)\right)<1 / 2^{n}-\varepsilon$; i.e., $\phi\left(t_{0}\right) \cap U \cap S \neq \varnothing$ where $S$ is the open $\left(1 / 2^{n}-\varepsilon\right)$-ball around $f_{n}\left(t_{0}\right)$. Since $\phi$ is l.s.c., $\phi(t) \cap U \cap S \neq \varnothing$ for all $t$ of some neighborhood $N_{1}$ of $t_{0}$. Since $f_{n}$ is continuous, we also have $d\left(f_{n}(t), f_{n}\left(t_{0}\right)\right)<\varepsilon$ for all $t$ in some neighborhood $N_{2}$ of $t_{0}$. If $t \in N_{1} \cap N_{2}$, then $\phi(t) \cap U \cap S \neq \varnothing$ and every point $y \in \phi(t) \cap U \cap S$ has the property that

$$
d\left(y, f_{n}(t)\right) \leqq d\left(y, f_{n}\left(t_{0}\right)\right)+d\left(f_{n}(t), f_{n}\left(t_{0}\right)\right)<1 / 2^{n} .
$$

Hence $\phi_{n+1}(t) \cap U \neq \varnothing$ for all $t \in N_{1} \cap N_{2}$, and $\phi_{n+1}$ is 1.s.c.

CoRollary (i). Let $C$ be a complete convex set without lines in a weak Hausdorff space, and let $d$ be the part metric on C. If $\phi$ is a lower semi-continuous function on $T$ to the nonempty closed convex subsets of one part $\Pi$ of $C$, then there exists a continuous selection function $f: T \rightarrow \Pi$ for $\phi$.

Proof. We mentioned already that $d$ is a convex metric on $\Pi$. By Theorem $9 I I$ is $d$-complete.

COROLlaRY (ii). Let $\phi$ be a lower semi-continuous mapping from 
a paracompact space $T$ to the nonempty closed convex subsets of one part $I$ of the cone $\mathscr{H}_{+}(X)$ of nonnegative Radon measures on a locally compact space $X$. Then there exists a continuous selection function for $\phi$. Let $f$ be any such function and $\mu \in \Pi$. Then $f$ has the form $f(t)=g_{t} \mu$, where $t \rightarrow g_{t}$ is a continuous mapping from $T$ into $L^{\infty}(\mu)$.

This follows immediately from Corollary (i) and Theorem 11.

6. Representing measures. Let $X$ be a compact Hausdorff space, and $C_{R}(X)$ be the space of all continuous real functions on $X$. A function space $B$ is a separating subspace of $C_{R}(X)$, containing the constant functions. We summarize here some facts from [1].

Let $T_{B}=\left\{F \in B^{\prime}: F \geqq 0, F(1)=1\right\}$. Then $T_{B}$ is convex and compact in the weak topology $\sigma\left(B^{\prime}, B\right)$ on $B^{\prime}$. The space $X$ can be embedded in $T_{B}$, and $B$ can be represented as the restrictions to $T_{B}$ of all weakly continuous affine functions on $B^{\prime}$. Gleason parts can be defined in $X$ in terms of the Harnack-type of inequality: $x \sim y$ if and only if there is $\alpha>1$ such that $\alpha^{-1} \leqq u(x) / u(y) \leqq \alpha$ for all $u>0$ in $B$. The parts of this relation are the intersections of $X$, considered as a subset of $T_{B}$, with the geometrically defined parts of $T_{B}$. The distance in parts in $X$ defined by $d(x, y)=\sup \{|\log u(x)-\log u(y)|: u>0\}$ is the same as the part metric in $T_{B}$.

For simplicity, by the boundary $\Gamma$ of $B$ we will mean the Choquet boundary if $X$ is metrizable (so that $\Gamma$ is a $G_{\hat{o}}$ ), or otherwise the Silov boundary (so $\Gamma$ is closed). A representing measure $\mu$ for $x \in X$ is a positive Radon measure supported by $\Gamma$ such that $u(x)=\int_{\Gamma} u d \mu$ for all $u \in B$. Let $\mathscr{C}_{+}^{1}=\mathscr{L}_{+}^{1}(X)$ be all positive probability measures on $X$ and let $\mathscr{l}_{x}$ be the representing measures for $x$. Clearly $\mathscr{H}_{x} \subset \mathscr{C l}_{+}^{1}$ for each $x$, since $1 \in B$.

If $A$ is a complex function algebra on $X$, and $B=\operatorname{Re} A$, then the spectrum $S_{A}$ can be considered a subset of $T_{B}$. The Gleason parts of $S_{A}$ are the intersections of $S_{A}$ with the parts of $T_{B}$. The metric on $S_{A}$ from the norm of $A^{\prime}$ gives the same topology as the part metric $[1,3]$. The Silov and Choquet boundaries for $A$ are the same as for $B$, and a measure represents a point for $A$ if and only if it represents the point for $B$. Therefore we will confine our discussion to the case of a real function space $B$, but our results will also apply to complex function algebras.

Harkova [9] has given an example of a function algebra (hence also a function space) where the complement of the Silov = Choquet boundary is one part $\Delta$, and it is not possible to pick representing measures $\mu_{x}$ for all $x \in \Delta$ so that all $\mu_{x}$ are in one part of $\mathscr{C}_{+}^{1}$. It is 
shown in [9], however, that if the space of orthogonal real measures is finite dimensional, then it is possible to pick representing measures so that points in one part of $X$ will have measures in one part of $\mathscr{L}_{+}^{1}$. We give here a condition based on our selection theorem which insures that if measures $\mu_{x}$ for points in one part can be chosen in one part of $-/_{+}^{1}$, then we can pick them so that $x \rightarrow \mu_{x}$ is a continuous mapping with respect to the two part metrics.

Lemma 5. The set $\mathscr{C l}_{x}$ of positive Radon measures on $X$ supported by the Choquet or Silov boundary $\Gamma$ which represent $x$ is convex and closed in the part metric of $\mathscr{H}_{+}^{1}(X)$.

Proof. The convexity is obvious. If $\mu_{n} \in \mathscr{C}_{x}$ and $d\left(\mu_{n}, \mu_{0}\right) \rightarrow 0$, then $\mu_{n}, \mu_{0}$ all are in one part of $\mathscr{L}_{+}^{1}$ (or $\mathscr{L}_{x}(X)$ ). Hence by Theorem $11 \mu_{n}=g_{n} \mu, \mu_{0}=g_{0} \mu$ for some $\mu$, where $g_{n}, g_{0} \in L^{\infty}(\mu)$, and $\left\|g_{n}-g_{0}\right\|_{\infty} \rightarrow 0$. It is clear that $\mu_{0}$ is supported by $\Gamma$. Since

$$
\left|\int u\left(g_{n}-g_{0}\right) d \mu\right| \leqq\|u\|\left\|g_{n}-g_{n}\right\|_{\infty} \rightarrow 0,
$$

$\int u g_{n} d \mu=u(x)=\int u g_{0} d \mu$ for all $u \in B$. Hence $\mu_{0} \in \mathscr{H}_{x}$.

Theorem 13. Let $B$ be a function space on $X$ with boundary $\Gamma$. Let $\triangle$ be a part of $X$ and suppose there is a part $\Pi_{\mu} \subset{ }_{-} Z_{+}^{1}$ such that $\mathscr{M}_{x} \cap \Pi_{\mu} \neq \varnothing$ for all $x \in \Delta$. If the mapping $x \rightarrow \mathscr{M}_{x} \cap \Pi_{\mu}$ is 1.s.c. with respect to the two part metrics then there is a continuous mapping $x \rightarrow \mu_{x}=g_{x} \mu$ on $\Delta$ into $\Pi_{\mu}$ such that $\mu_{x} \in \mathscr{l l}_{x}$ for each $x$. Consequently, the mapping $x \rightarrow g_{x}$ is d-continuous from $\Delta$ into $L^{\infty}(\mu)$.

Proof. Since each $\mathscr{C l}_{x}$ is closed in $\mathscr{Z}_{+}^{1}$, and each part $\Pi_{\mu}$ is $d$ closed, the sets $\mathscr{C}_{+} \subset P_{\mu}$ are $d$-closed, and clearly also convex. The metric space $(\Delta, d)$ is paracompact. The part metric in $\mathscr{C}_{+}^{1}$ is complete, since $\mathscr{C}_{+}^{1}$ is compact in the vague topology. The theorem is now immediate from Theorem 12, Corollary (i).

We remark that the part metric in a part $\Delta$ of $X$ gives the relativization to $\Delta$ of the given compact topology of $X$ if the set

$$
B^{+}\left(z_{0}\right)=\left\{u \mid \Delta: u \in B, u>0, u\left(z_{0}\right)=1\right\} \quad\left(z_{0} \in \Delta\right)
$$

is equicontinuous [2, Th. 3], [10, Lemma 1]. This is the case for example for spaces $B$ of functions continuous on the closure of a bounded domain in $R^{n}$ and harmonic on the interior. In such a case the continuity of the mapping $x \rightarrow \mu_{x}=g_{x} \mu$ with respect to the part metric is the same as continuity with respect to the given Euclidean 
topology in $X$ and the part metric topology in $\mathscr{C l}_{++}^{1}$ (or the $L^{\infty}(\mu)$ topology.)

Added in proof. The part metric is similar to the metric of Cayley-Klein (cf. W. BLASCHKE, Projektive Geometrie, Basel (1954), p. 80). A. C. Thompson has recently used the part metric in the form given here to simplify some proofs of Birkhoff and Samelson (On certain contraction mappings in a partially ordered vector space, P.A.M.S. vol. 14 (1963), 438-443).

\section{REFERENCES}

1. H. S. Bear, A geometric characterization of Gleason parts, Proc. Amer. Math. Soc. 16 (1965), 407-412.

2. H. S. Bear and Bertram Walsh, Integral kernel for one-part function spaces, Pacific J. Math. 23 (1967), 209-215.

3. H. S. Bear and M. L. Weiss, An intrinsic metric for parts, Proc. Amer. Math. Soc. 18 (1967), 812-817.

4. N. Bourbaki, Espaces vectoriels topologiques, Chap. 1-2 (2nd edition), Act. Sci. et Ind. 1189, Paris, 1966.

5. - Intégration, Chap. 1-4, Act. Sci. et Ind. 1175, Paris, 1952.

6. Intégration, Chap. 5, Act. Sci et Ind. 1244, Paris, 1956.

7. G. Choquet, Ensembles et cônes convexes faiblement complets, C. R. Académie Sciences 254 (1962), 1908-1910; 2123-2125.

8. A. M. Gleason, Function algebras, Seminar on analytic functions, vol. 2, Institute for Advanced Study, Princeton, N. J., 1957.

9. N. V. Harkova, Generalized Poisson formula (Russian), Vestnik Moskov. Univ., Ser. I Mat. Meh. 22 (1967), 25-30.

10. J. Köhn, Die Harnacksche Metrik in der Theorie der harmonischen Funktionen, Math. Zeit. 91 (1966), 50-64.

11. E. Michael, Selected selection theorems, Amer. Math. Monthly 43 (1956), 233-238.

12. Continuous selections I, Ann. of Math. 63 (1956), 361-381.

Received November 13, 1968. This is a revised form of a paper submitted by H. S. Bear on March 19, 1968, This author was supported in part by Grant GP7681 of the National Science Foundation.

UNIVERSITÄT ERLANGEN-NÜRNBERG

New Mexico State University

AND

UNIVERSITY OF HAWAII 



\section{PACIFIC JOURNAL OF MATHEMATICS}

\section{EDITORS}

H. ROYDEN

Stanford University

Stanford, California

Richard Pierce

University of Washington

Seattle, Washington 98105
J. DugundJI

Department of Mathematics

University of Southern California

Los Angeles, California 90007

BASIL GORDON

University of California

Los Angeles, California 90024

\section{ASSOCIATE EDITORS}

E. F. BECKENBACH

B. H. NeUmanN

F. WOLF

K. YOSHIDA

\section{SUPPORTING INSTITUTIONS}

UNIVERSITY OF BRITISH COLUMBIA

CALIFORNIA INSTITUTE OF TECHNOLOGY

UNIVERSITY OF CALIFORNIA

MONTANA STATE UNIVERSITY

UNIVERSITY OF NEVADA

NEW MEXICO STATE UNIVERSITY

OREGON STATE UNIVERSITY

UNIVERSITY OF OREGON

OSAKA UNIVERSITY

UNIVERSITY OF SOUTHERN CALIFORNIA
STANFORD UNIVERSITY

UNIVERSITY OF TOKYO

UNIVERSITY OF UTAH

WASHINGTON STATE UNIVERSITY

UNIVERSITY OF WASHINGTON

$* * \quad * \quad *$
AMERICAN MATHEMATICAL SOCIETY
CHEVRON RESEARCH CORPORATION
TRW SYSTEMS
NAVAL WEAPONS CENTER




\section{Pacific Journal of Mathematics}

Vol. 30, No. $1 \quad$ September, 1969

William Wells Adams, Simultaneous diophantine approximations and cubic irrationals ..................................... 1

Heinz Bauer and Herbert Stanley Bear, Jr., The part metric in convex

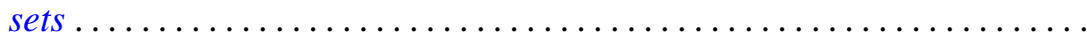

L. Carlitz, A note on exponential sums ...................... 35

Vasily Cateforis, On regular self-injective rings ................. 39

Franz Harpain and Maurice Sion, A representation theorem for measures on infinite dimensional spaces ......................... 47

Richard Earl Hodel, Sum theorems for topological spaces .............. 59

Carl Groos Jockusch, Jr. and Thomas Graham McLaughlin, Countable

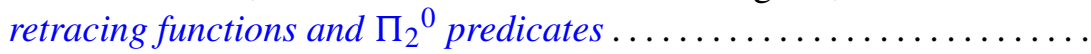

Bjarni Jónsson and George Stephen Monk, Representations of primary

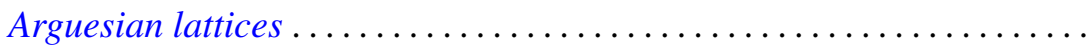

Virginia E. Walsh Knight, A continuous partial order for Peano continua...................................... 141

Kjeld Laursen, Ideal structure in generalized group algebras ........... 155

G. S. Monk, Desargues' law and the representation of primary lattices . . . 175

Hussain Sayid Nur, Singular perturbation of linear partial differential equation with constant coefficients ..........................

Richard Paul Osborne and J. L. Stern, Covering manifolds with cells ... 201

Keith Lowell Phillips and Mitchell Herbert Taibleson, Singular integrals in several variables over a local field...

James Reaves Smith, Local domains with topologically $T$-nilpotent radical....

Donald Platte Squier, Elliptic differential equations with discontinuous coefficients .................................

Tae-il Suh, Algebras formed by the Zorn vector matrix...

Earl J. Taft, Ideals in admissible algebras . .................... 259

Jun Tomiyama, On the tensor products of von Neumann algebras........ 263

David Bertram Wales, Uniqueness of the graph of a rank three group ..... 271

Charles Robert Warner and Robert James Whitley, A characterization of regular maximal ideals ......................... 